Proceedings of the 12th International Symposium UFPS, Vilnius, Lithuania 2004

\title{
Ultrafast Bimolecular Recombination in Nanocrystalline Hydrogenated Silicon
}

\author{
N. Nekrašas ${ }^{a}$, G. Sliaužys ${ }^{a}$, G. Juška ${ }^{a, *}$, K. Arlauskas ${ }^{a}$, \\ J. STUCHLIK ${ }^{b}$ AND J. KOČKA ${ }^{b}$ \\ ${ }^{a}$ Department of Solid State Electronics, Vilnius University \\ Saulètekio 9, III k., 01513 Vilnius, Lithuania \\ ${ }^{b}$ Institute of Physics, Academy of Science \\ Cukrovarnicka 10, 16253 Prague, Czech Republic
}

\begin{abstract}
In the multilayers of hydrogenated nanocrystalline and amorphous silicon bimolecular recombination coefficient can be reduced in half, while in low-temperature hydrogenated nanocrystalline silicon samples it can be reduced by one order of magnitude. The similarity of the activation energies of both the bimolecular recombination $(B)$ and the Langevin-type recombination $\left(B_{\mathrm{L}}\right)$ coefficients point to decisive role of tunneling in processes of meeting of electrons and holes, although the ratio $B / B_{\mathrm{L}}<0.01$.
\end{abstract}

PACS numbers: 73.50.Gr, 72.20.Jv, 84.60.Jt

\section{Introduction}

The nonradiative bimolecular recombination coefficient $(B)$ of charge carriers is one of the testing parameters for the solar cells of low mobility materials. In low mobility materials, where the hopping distance of charge carriers is shorter than the Coulomb radius, bimolecular recombination is caused by the meeting probability of hole and electron in coordinate space (Langevin-type recombination coefficient $B_{\mathrm{L}}=e \mu / \varepsilon \varepsilon_{0}$ where the symbols have their usual meaning). However, the bimolecular recombination can be decreased by intrinsic random potential, which separates paths of electrons and holes and reduces their chances to meet. The latter causes that in hydrogenated amorphous silicon (a-Si:H) $B$ is by twothree orders lower than $B_{\mathrm{L}}$ [1]. Moreover, the energy released during bimolecular recombination causes degradation of the solar conversion efficiency of amorphous silicon-based cells [2].

The aim of this research is to investigate influence of layers structure on bimolecular recombination in nanocrystalline silicon layers (nc-Si:H), deposited on substrates of various temperature during deposition and in multilayers of different nc-Si:H/a-Si:H compositions. The way of improvement of nc-Si:H photoelectric features is discussed too.

*corresponding author; e-mail: gytis.juska@ff.vu.lt 


\section{Method}

The photoelectric method of estimation of $B$ is based on the measurement of the extraction time $\left(t_{\mathrm{e}}\right)$ and extracted residual amount of charge carriers $\left(Q_{\mathrm{e}}\right)$, generated by a short and intensive pulse of light. The $B$ value is calculated from saturation values of $t_{\mathrm{e}}$ and $Q_{\mathrm{e}}$ [1]. The theory of the latter method was revised and adapted to the case of a thin sample, namely, to case $\alpha d \approx 1$, where $\alpha$ is the absorption coefficient and $d$ is the thickness of the sample. $d \approx 1 \mu \mathrm{m}$ is typical thickness of a-Si:H and nc-Si:H solar cells.

At the very initial moment of time, $t=0$, when the sample is illuminated by a short pulse of light of intensity $L$, the charge carriers are photogenerated and their recombination and extraction by extrinsic electric field begins. When $L$ is sufficiently high, recombination of photogenerated charge carriers of density $n$ can be described by the equation $\mathrm{d} n / \mathrm{d} t=-B n^{2}$. Taking into account the initial condition $n(x, 0)=L \alpha \exp (-\alpha x)$, we can obtain the solution of this equation

$$
n(x, t)=\left[\frac{1}{L \alpha \exp (-\alpha x)}+B t\right]^{-1} .
$$

In the considered case the extraction current is limited by load resistance $\left(R_{\mathrm{L}}\right)$ while in the case of $\alpha d \gg 1$ it is limited by space charge. As mentioned before, the current reduces the width of the charge carrier's reservoir, but it has no influence on the carrier density $(n)$ in the reservoir, where electric field is absent. We can choose $B_{\mathrm{L}}$ low enough to extract all charge carriers before the monomolecular recombination reveals itself, so (1) retains its validity till the extraction time $\left(t_{\mathrm{e}}\right)$.

The amount $N$ of residual (non-recombinated) charge carriers can be obtained by integration of (1) over the thickness of the sample, $N(t)=d / B t$. Of course, the whole reservoir of charge $(N)$ will be extracted, mostly during $t_{\mathrm{e}}$, thus

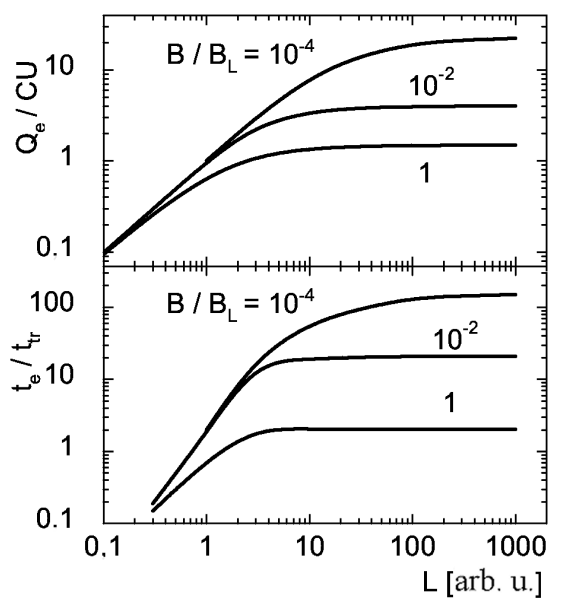

Fig. 1. Numerical simulation results of the dependences of the normalized extracted charge carriers amount $Q_{\mathrm{e}}$ and their extraction time $t_{\mathrm{e}}$ on the light pulse intensity $L$. 
we have (when $L \rightarrow \infty$ )

$$
N\left(t_{\mathrm{e}}\right)=\frac{d}{B t_{\mathrm{e}}}=\frac{Q_{\mathrm{e}}}{e}=\frac{1}{e} \int_{0}^{t_{\mathrm{e}}} j(t) \mathrm{d} t .
$$

The latter formula could be used to estimate $B$ experimentally.

One can see from Eq. (2) that $N(t)$ is independent of $L$ in the case of bimolecular recombination. Therefore, increasing $L$ leads to the saturation of $t_{\mathrm{e}}$ and $Q_{\mathrm{e}}$, while it is known that in the case of the monomolecular recombination $t_{\mathrm{e}} \sim \ln (\mathrm{L})[1]$. The differences of mentioned $t_{\mathrm{e}}$ and $Q_{\mathrm{e}}$ dependencies on $L$ enables us to distinguish between monomolecular and bimolecular recombination cases.

Numerical modeling results demonstrate saturation of $t_{\mathrm{e}}$ and $Q_{\mathrm{e}}$ (see Fig. 1) verifying possibility of $B$ estimation from Eq. (3).

\section{Experiment}

We investigated two series of samples. Both series were prepared by 13.56 MHz RF plasma enhanced chemical vapor deposition (CVD) on the Corning glass substrate covered by the ITO electrode. The first set of samples was prepared with 3 and $4.5 \%$ silane in $\mathrm{H}_{2}$ dilution and substrate temperature was $60-250^{\circ} \mathrm{C}$. The second set, called multilayers, was prepared at $250^{\circ} \mathrm{C}$ by changing the $\mathrm{H}_{2}$ dilution from $8 \% \mathrm{SiH}_{4}$ (for a-Si:H sublayer deposition) to $4.5 \% \mathrm{SiH}_{4}$ in $\mathrm{SiH}_{4}+\mathrm{H}_{2}$ mixtures (for nc-Si:H sublayer). Using the known growth rates of a-Si:H and nc-Si:H, the alternating deposition intervals were adjusted to achieve a projected crystalline fraction $X_{\mathrm{c}}=d_{\mathrm{nc}} /\left(d_{\mathrm{nc}}+d_{\mathrm{a}}\right)$, where $d_{\mathrm{nc}}$ and $d_{\mathrm{a}}$ are thicknesses of the nanocrystalline and amorphous sublayers, respectively. Thickness of both kinds of samples was approximately $1 \mu \mathrm{m}$. The top semitransparent Al electrodes were prepared by vacuum evaporation.

Photoexcitation was provided by $20 \mathrm{ps}$ pulses of a $540 \mathrm{~nm}$ Nd:YAG laser through the bottom ITO electrode. At this wavelength absorption coefficients of nc-Si:H and a-Si:H are $2 \times 10^{4} \mathrm{~cm}^{-1}$ and $9 \times 10^{4} \mathrm{~cm}^{-1}$, respectively.

\section{Results and discussion}

The level of charge carriers transport dispersion may be estimated quantitatively by CELIV method as a ratio $t_{1 / 2} / t_{\max }[3]$. Figure 2 demonstrates that the decrease in the temperature of the substrate during deposition leads to increase in $t_{1 / 2} / t_{\max }$, and, thus, to the increase in influence of an intrinsic random potential. This causes the decrease in $B$, because the intrinsic random potential separates photogenerated charge carriers of opposite sign. The values of $B$, estimated as a function of the substrate temperature are shown in Fig. 2, too. As one can see, the value of $B$ is about 100 times lower than $B_{\mathrm{L}} \approx 1.5 \times 10^{-7} \mathrm{~cm}^{3} / \mathrm{s}$ for the samples of the pure nc-Si:H, deposited at conventional temperature $\left(250^{\circ} \mathrm{C}\right)$, and decreases with lowering the substrate's temperature during deposition. Due to dispersive transport the shape of photocurrent transients becomes featureless, so, it is more 

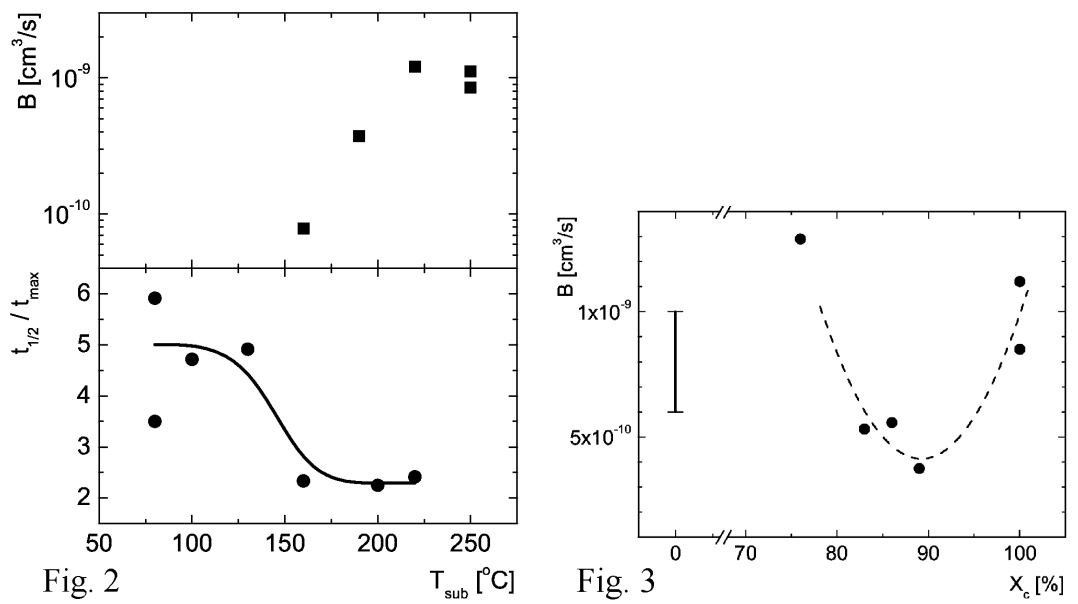

Fig. 2. Bimolecular recombination coefficient $B$ and dispersion parameter $t_{1 / 2} / t_{\max }$ dependences on the substrate temperature during deposition $T_{\text {sub }}$.

Fig. 3. Bimolecular recombination coefficient $B$ dependence on the projected crystalline fraction $X_{\mathrm{c}}$.

difficult to estimate $Q_{\mathrm{e}}$ and $t_{\mathrm{e}}$ in the low-temperature samples and even impossible in the case when $T_{\text {sub }}<150^{\circ} \mathrm{C}$.

The low activation energy $(0.046 \mathrm{eV})$ of $B$ is similar to that of $B_{\mathrm{L}}$ and may be interpreted as being due to a decisive role of tunneling, which increases the meeting probability of electron and hole.

In Fig. 3 the results of estimation of $B$ in the multilayers are presented. As can be seen, the $B$ varies in a relatively narrow range and has a minimum at $X_{\mathrm{c}}=90 \%$. Contrary to our expectations, no significant decrease in $B$ was observed. The latter can be explained by the fact that changing conditions during deposition of multilayer does not completely stop growth of the crystallites. Therefore, the structure of multilayer is more like the blend.

\section{Acknowledgment}

Financial support from Lithuanian VMSF grant No. P-17/01 is acknowledged.

\section{References}

[1] G. Juška, M. Viliūnas, K. Arlauskas, J. Kočka, Phys. Rev. B 51, 16668 (1995).

[2] G. Juška, M. Viliūnas, K. Arlauskas, J. Stuchlik, J. Kočka, Phys. Status Solidi A 171, 539 (1999).

[3] G. Juška, K. Arlauskas, N. Nekrašas, G. Sliaužys, Lith. J. Phys. 43, 258 (2003). 